\title{
Role of N6-methyladenosine Modification in Cardiac Remodeling
}

\author{
ManTing Choy ${ }^{1,2 \dagger}$, Ruicong Xue ${ }^{1,2 \dagger}$, Yuzhong $W_{u^{1,2}}$, Wendong Fan ${ }^{1,2}$, Yugang Dong ${ }^{1,2}$ and \\ Chen Liu $^{1,2 *}$
}

${ }^{1}$ Department of Cardiology, The First Affiliated Hospital of Sun Yat-sen University, Guangzhou, China, ${ }^{2}$ National Health Commission (NHC) Key Laboratory of Assisted Circulation, Sun Yat-sen University, Guangzhou, China

OPEN ACCESS

Edited by:

Kun Wang,

Qingdao University, China

Reviewed by:

Yuhui Zhang,

Chinese Academy of Medical

Sciences and Peking Union Medical

College, China

Luyu Zhou,

Hunan University, China

*Correspondence:

Chen Liu

liuch75@mail.sysu.edu.cn

†These authors share first authorship

Specialty section:

This article was submitted to

General Cardiovascular Medicine,

a section of the journal

Frontiers in Cardiovascular Medicine

Received: 23 September 2021

Accepted: 17 January 2022

Published: 10 February 2022

Citation:

Choy M, Xue R, Wu Y, Fan W, Dong Y and Liu C (2022) Role of

N6-methyladenosine Modification in

Cardiac Remodeling.

Front. Cardiovasc. Med. 9:774627.

doi: 10.3389/fcrm.2022.774627
Cardiac remodeling is the critical process in heart failure due to many cardiovascular diseases including myocardial infarction, hypertension, cardiovascular disease and cardiomyopathy. However, treatments for heart failure focusing on cardiac remodeling show relatively limited effectiveness. In recent decades, epitranscriptomic modifications were found abundantly present throughout the progression of cardiac remodeling, and numerous types of biochemical modifications were identified. m6A modification is the methylation of the adenosine base at the nitrogen- 6 position, and dysregulation of m6A modification has been implicated in a wide range of diseases. However, function of m6A modifications still remain largely unknown in cardiac diseases, especially cardiac remodeling. LncRNAs are also shown to play a vital role in the pathophysiology of cardiac remodeling and heart failure. The crosstalk between IncRNAs and m6A modification provides a novel prospective for exploring possible regulatory mechanism and therapeutic targets of cardiac remodeling. This review summarizes the role of m6A modification in cardiac remodeling in the current researches.

Keywords: cardiac remodeling, m6A modification, IncRNAs, heart failure, epigenetic modifications

\section{INTRODUCTION}

Cardiac remodeling is a physiologic and pathologic condition that may occur after various cardiovascular diseases including myocardial infarction, hypertension, cardiovascular disease and cardiomyopathy (1). Cardiac remodeling is a dynamic process in which the heart changes in size, mass, geometry and function in response to mechanical stress such as pressure or volume overload, neurohormones or cytokines in order to maintain hemodynamic homeostasis. When cardiac remodeling progresses, the transition from possible adaptive to maladaptive remodeling occurs, which may likely to establish heart failure (HF) eventually. Treatment for cardiac remodeling have been focused on neurohormonal inhibition such as renin-angiotensin-aldosterone system (RAAS) or sympathetic nervous systems (SNS) inhibition, but the proportion of poor prognosis of HF remained high. As a result, understanding the underlying pathophysiological processes involved in cardiac remodeling is critical for developing novel therapeutic strategies.

Epigenetic modifications are abundantly present throughout the physiological processes of life. It is characterized by the modification in temporal and spatial expression patterns of chromatins and genes driven by particular enzymes, without changing the nucleotide sequence of DNA and subsequent functional alterations of heritable gene $(2,3)$. RNA modification, also term epitranscriptomic modification, is one of these epigenetic modifications. So far, at least 170 types of RNA modifications have been discovered (4). N6-methyladenosine (m6A) is one of the abundant 
mRNA modifications in almost all eukaryotes. It refers to methylation of the adenosine base at the nitrogen- 6 position (5). It is written by a complex of m6A methyltransferase, erased by m6A demethylase, and read by m6A binding proteins. m6A modifications govern RNA processing, including splicing, nuclear exports, RNA stability and translation, by recognizing $\mathrm{m} 6 \mathrm{~A}$ binding proteins (6). $\mathrm{m} 6 \mathrm{~A}$ has been proved to be involved in the regulation of a wide variety of pathological processes including obesity, metabolic disease and carcinogenesis. For example, one of the m6A component, the RNA methyltransferase METTL3, was discovered to promote proliferation and invasion of human lung cancer cells (7). As for cardiovascular disorders, continuous dynamic control of m6A have been shown to play a critical role as well. METTL14, another methyltransferase of RNA, enhanced the m6A modification of pri-miR-19a and promoted mature miR-19a processing to increasing atherosclerosis vascular endothelial cells proliferation and invasion (8). At the moment, researches of m6 A modification in the field of heart failure and cardiac remodeling are still in their initial phases. Zhang et al. revealed the different expression profiles of $\mathrm{m} 6 \mathrm{~A}$ regulators in heart failure with preserved ejection fraction (HFpEF) patients in combination with a clinical case-control study and animal experiment, and highlighted the relationship between $\mathrm{m} 6 \mathrm{~A}$ modification and the risks of $\mathrm{HFpEF}$ (9). Given the comprehensive regulation of m6A modification in diseases, much remains to be explored in cardiac remodeling and heart failure.

Long non-coding RNAs (lncRNAs) are non-protein coding transcripts with a length more than 200 nucleotides. LncRNAs could govern the cellular processes, such as cell cycles regulation, differentiation through diverse mechanisms like transcription, translation, splicing, etc, in various disease states (10). Emerging data have shown that lncRNAs play a key role in the pathogenesis of cardiac remodeling and heart failure (11). Many mature lncRNAs are modified after transcription, and numerous types of biochemical modifications were found in lncRNAs (12). The relationship between $\operatorname{lncRNAs}$ and m6A modifications still remain largely unknown, provides a fresh viewpoint for exploring the possible regulatory mechanism and suggests that m6A modification and lncRNAs interplay might be significant treatment targets for various diseases (13). This review provides an overview of recent advances in $\mathrm{m} 6 \mathrm{~A}$ modifications and gives

\footnotetext{
Abbreviations: m6A, N6-methyladenosine; LncRNAs, long non-coding RNAs; HF, heart failure; RAAS, renin-angiotensin-aldosterone system; SNS, sympathetic nervous systems; HFpEF, heart failure with preserved ejection fraction; METTL3, Methyltransferase like-3; METTL14, Methyltransferase like-14; WTAP, Wilms' tumor 1-associated protein; RBM15, RNA Binding Motif Protein 15; ZC3H13, Zinc Finger CCCH-Type Containing 13; RBM14, RNA Binding Motif Protein 14; FTO, fat mass and obesity-associated gene; ALKBH5, AlkB Homolog 5; YTHDF/YTHDC, YT521-B homology (YTH) domain family of proteins; IGF2BPs, insulin-like growth factor 2 mRNA-binding protein; HNRNPA2B1, heterogeneous nuclear ribonucleoproteins A2/B1; eIF3, eukaryotic initiation factor 3; ECM, extracellular matrix; ECs, endothelial cells; VSMCs, vascular smooth muscle cells; ADSCs, adipose-derived stem cells; $\mathrm{H} / \mathrm{R}$, hypoxia/reoxygenation; I/R, ischemia/reperfusion; Mhrt, myosin heavy chain associated RNA transcript; XIST, X-inactive specific transcript; MALAT1, metastasis-associated lung adenocarcinoma transcript 1; MAPKs, mitogenactivated protein kinases.
}

an updated outline of the association between m6A modification and cardiac remodeling, and provide an insight into potential molecular biomarkers associated with the m6A modification of lncRNAs and therapeutic targets in cardiac remodeling.

\section{MACHINERY AND BIOLOGICAL ROLES OF M6A MODIFICATION}

m6A modification is found throughout species in evolutionary patterns, having a consensus sequence of the m6A center site. Their critical roles in epigenetics and physiological connections to numerous human diseases have given them a huge scientific and medical attention. m6A modification is a dynamic and reversible posttranscriptional modification process which was implemented in three distinct kinds of protein complexes ("writer" and "reader" and "eraser" proteins) and can alter important biological processes by adding, removing, or preferring identify the m6A sites (Figure 1).

\section{Writer}

Methyltransferase (m6A "writers") are protein components that catalyze m6A methylation of RNAs. m6A modification is achieved by a large methyltransferase complex (MTC). The m6A MTC mainly consists of the methyltransferase-like proteins, such as METTL3 and METTL14, and various protein factors indispensable for their proper catalysis, including WTAP, RBM15, ZC3H13, and RBM14. METTL3 is responsible for the installation of m6A on RNA. According to a phylogenetic study, METTL14 is a homolog of METTL3, with $43 \%$ similarity. The METTL3/METTL14 form a stable heterodimer core complex exhibits far more m6A methyltransferase activity than either component alone. The METTL3/METTL14 complex preferentially methylates RNA on sites of $\mathrm{RRACH}(\mathrm{R}=$ $\mathrm{A} / \mathrm{G}, \mathrm{H}=\mathrm{A} / \mathrm{U} / \mathrm{C})$ in vitro, in accordance with the $\mathrm{RRACH}$ motif distribution of m6A transcriptome-wide (14). WTAP, as the scaffold protein of m6A-MTC, exhibits an influence on the METTL3/METTL14 compound structure and substrate composition (15). Additionally, several proteins have been shown to involved in substrate binding, catalytic efficiency, stability, and localization in addition to the core of m6A complex. RBM15, ZC3H13, HAKAI, and VIRMA, for example, have been identified as WTAP components, and this association offers a scaffold for METTL3/METTL14 recruitment for methylation (16).

\section{Eraser}

Demethylases (m6A "erasers") are enzymes that remove the methyl group from RNA. m6A is one of the few intrinsic RNA modifications that may be reversed. The identification of RNA demethylase FTO, was the crucial breakthrough that revived the m6A function. FTO catalyzes oxidative demethylation of $\mathrm{m} 6 \mathrm{~A}$ in an $\mathrm{Fe}(\mathrm{II})$ - and $\alpha$-KG-dependent manner which preferentially bind to pre-mRNA strands in intronic regions, in the proximity of alternatively spliced exons and poly(A) sites (17). FTO is a member of the ALKB family. Another family member, ALKBH5, has also been discovered as a demethylase. Both ALKBH5 and FTO are confined to nucleus and located by nuclear speckles, which show the splicing effects of methylation (18). Instead of 


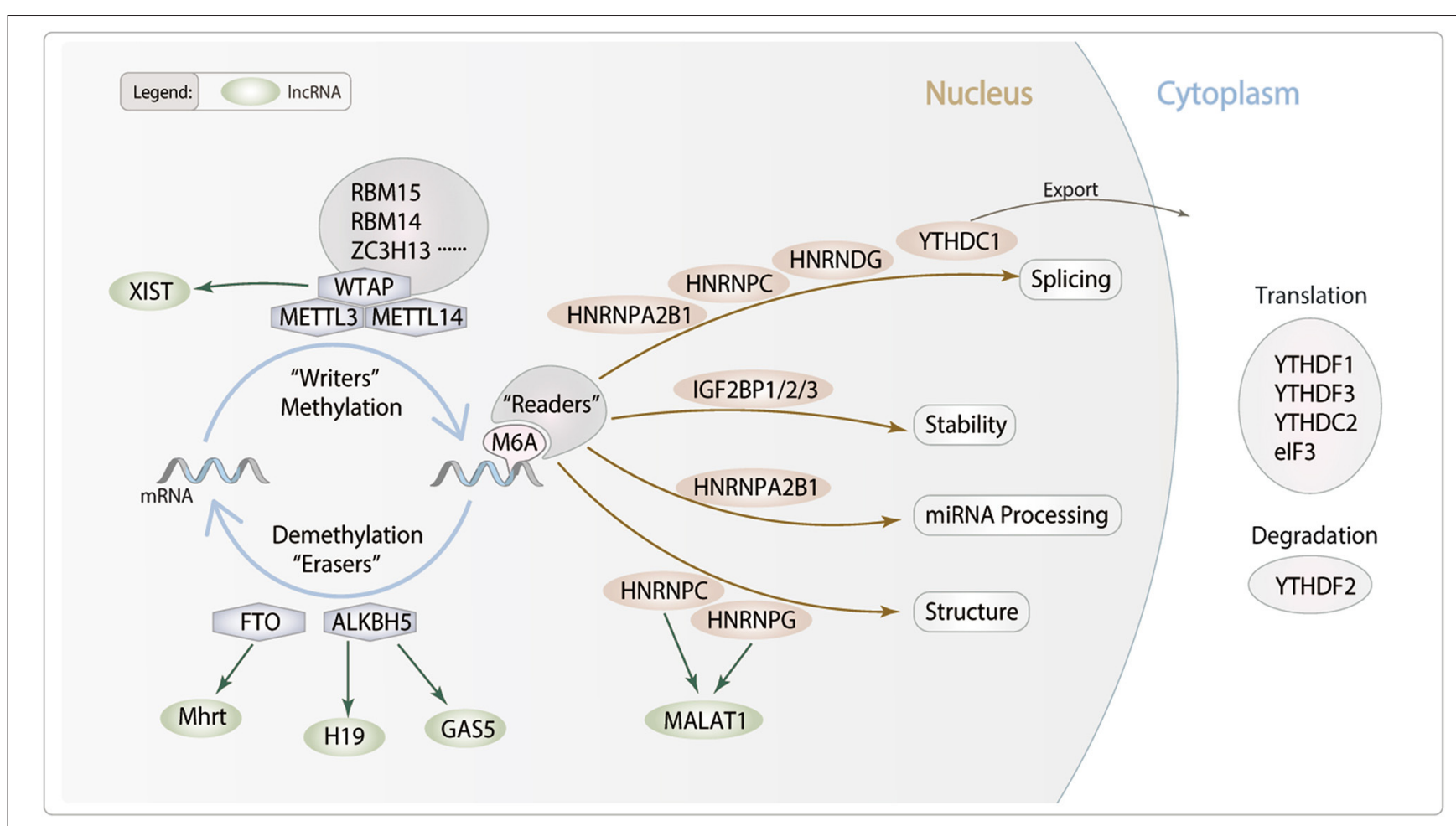

FIGURE 1 | Potential role of IncRNA m6A modification in cardiac remodeling. m6A is deposited by "Writers" (METTL3/14, WTAP, RBM14/15 and ZC3H13), removed by "Erasers" (FTO and ALKBH5), and recognized by "Readers" (YTHDC1/2, YTHDF1/2/3, IGF2BP1/2/3, HNRNPA2B1, HNRNPC, HNRNPG and elF3). m6A modifications can regulate RNA processing, including splicing, nuclear exports, stability and translation. We hypothesis IncRNA m6A modification which may modulate particular pathophysiological process of cardiac remodeling.

the FTO oxidative demethylation, ALKBH5 catalytic reaction directly removes the methyl group from the m6A methylated adenosine (19). ALKBH5 found as m6A mRNA demethylase employing $\mathrm{Fe}^{2+}$ and 2OG cofactor, which work together to remove the methyl group in $\mathrm{m} 6 \mathrm{~A}$ containing substrates.

\section{Reader}

The biological function of $\mathrm{m} 6 \mathrm{~A}$ modification is regulated by m6A-binding proteins, also known as the "readers," including YTHDF1/2/3, YTHDC1/2, IGF2BP1/2/3, HNRNPA2B1 and eIF3. Reader proteins that act as functional mediators, selectively identity target m6A-modified mRNA and regulate varies of RNA metabolism processes such as RNA splicing, transport, translocation, translation and degradation (20). YTHDC1 is a nuclear protein involved in gene splicing. However, YTHDF1-3 are cytoplasmic m6A readers. YTHDF1 and YTHDF3 collaborate to influence the translation of m6Acontaining mRNAs, whereas YTHDF2 speeds up mRNA decay and YTHDC1 influences the nuclear processing of its targets (21). Moreover, a number of recent papers claim to have discovered the presence of additional sorts of readers. IGF2BPs (IGF2BP1-3), HNRNPA2B1 and eIF3 can affect the splicing, translation, stability and degradation of mRNA (21). IGF2BPs belong to a conserved family of RNA-binding, and associate with target mRNAs in cytoplasmic ribonucleoprotein complexes, and enhance the stability and storage of their target mRNAs, therefore regulating the output of gene expression (22). HNRNPA2B1 can directly binds nuclear transcripts to induces alternative splicing effects and boosting microRNA processing (23). eIF3 plays an important role in recruitment of the pre-initiation complex PIC to mRNA. Moreover, YTHDF1 relocates from the cytoplasm to the nucleus and regulates translation may depends on interaction with eIF3 (24).

\section{M6A METHYLATION IN CARDIAC REMODELING}

Cardiac remodeling involves almost all the cell types in the heart including cardiomyocytes, cardiac fibroblasts, vascular smooth muscle cells and vascular endothelial cells. Remodeling encompasses cellular changes including cardiomyocytes hypertrophy, necrosis, apoptosis, vascular differentiation and fibroblast proliferation. Recently, it was discovered that m6A methylation plays an important role in mediating significant structural alterations in the failing heart. Researchers observed that the overall level of m6A modification of the transcripts in the healthy mouse and human heart is increased by using next-generation sequencing. Moreover, the changes in m6A methylation exceeded changes in gene expression with the course of heart failure in both mice and humans (25). 
TABLE 1 | Role of m6A modification in cardiac remodeling.

\begin{tabular}{|c|c|c|c|c|c|}
\hline $\begin{array}{l}\text { Cardiac } \\
\text { remodeling }\end{array}$ & m6A regulators & m6A levels & Cell (tissue) types & Main functions and mechanisms & References \\
\hline \multirow[t]{3}{*}{$\begin{array}{l}\text { Cardiomyocytes } \\
\text { hypertrophy }\end{array}$} & $\begin{array}{l}\text { METTL3 } \\
\text { upregulated }\end{array}$ & Increased & Cardiomyocytes under hypertrophic conditions. & $\begin{array}{l}\text { METTL3 promotes the expression of MAPKs } \\
\text { family in cardiomyocytes. }\end{array}$ & (27) \\
\hline & $\begin{array}{l}\text { METTL3 } \\
\text { upregulated }\end{array}$ & Increased & Human failing myocardium. & $\begin{array}{l}\mathrm{m} 6 \mathrm{~A} \text { regulates translational efficiency by } \\
\text { affecting transcript stability. }\end{array}$ & (28) \\
\hline & FTO upregulated & Decreased & Cardiomyocytes by leptin stimulation. & $\begin{array}{l}\text { FTO upregulation via JAK2/STAT3-dependent } \\
\text { CUX1 upregulation. }\end{array}$ & (29) \\
\hline $\begin{array}{l}\text { Cardiomyocytes } \\
\text { death }\end{array}$ & $\begin{array}{l}\text { METTL3 upregulate } \\
\text { ALKBH5 } \\
\text { downregulated }\end{array}$ & d Increased & $\begin{array}{l}\mathrm{H} / \mathrm{R} \text {-treated cardiomyocytes and I/R-treated } \\
\text { mice heart. }\end{array}$ & $\begin{array}{l}\text { METTL3 enhances the binding of TFEB to } \\
\text { HNRNPD, which decreases TFEB expression, } \\
\text { thereby impairing autophagic flux and } \\
\text { enhancing apoptosis. ALKBH5 exerts the } \\
\text { opposite effects. }\end{array}$ & (32) \\
\hline & $\begin{array}{l}\text { METTL3 } \\
\text { upregulated }\end{array}$ & Increased & Mice hearts. & $\begin{array}{l}\text { METTL3 overexpression following TAC } \\
\text { operation decreases fibrosis and collagen } \\
\text { transcription. }\end{array}$ & (28) \\
\hline & FTO upregulated & Decreased & Murine MI hearts. & Reduces scar size. & (34) \\
\hline \multirow[t]{2}{*}{$\begin{array}{l}\text { Vascular } \\
\text { remodeling }\end{array}$} & $\begin{array}{l}\text { METTL3 } \\
\text { upregulated }\end{array}$ & Increased & $\begin{array}{l}\text { ADSCs undergoing VSMCs differentiation } \\
\text { induction. }\end{array}$ & $\begin{array}{l}\text { Stimulates the differentiation of ADSCs into } \\
\text { vascular VSMCs and regulates the secretion of } \\
\text { VEGF, HGF, TGF- } \beta \text {, GM-CSF, bFGF, and } \\
\text { SDF-1. }\end{array}$ & (38) \\
\hline & FTO upregulated & Decreased & Murine Ml hearts. & $\begin{array}{l}\text { Reduces cardiac fibrosis (decreases of scar } \\
\text { size\%) and increases angiogenesis (higher } \\
\text { number of CD31- positive cells). }\end{array}$ & (34) \\
\hline
\end{tabular}

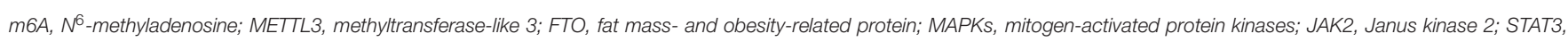
signal transduction and activator of transcription 3; CUX1. Cut Like Homeobox 1; MI, myocardial infarction; TFEB, Transcription Factor EB; H/R, hypoxia/reoxygenation; I/R,

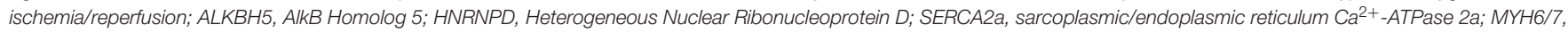

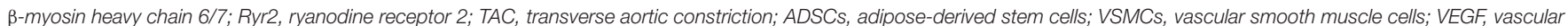

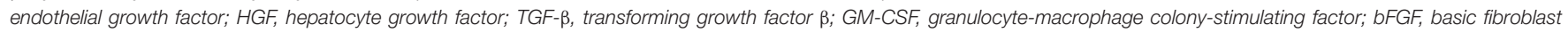

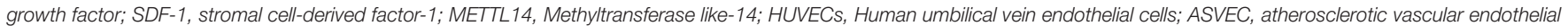
cells; FOXO1, forkhead box 01; ECs, endothelial cells.

\section{Cardiomyocytes Hypertrophy}

Cardiac hypertrophy is one of the predominant components of cardiac remodeling. During the early stages of stress, cardiac hypertrophy serves as a compensatory mechanism occurred in cardiomyocytes, manifested as myocytes hypertrophy, and progressive thickening of the ventricular wall. Sustained pathological hypertrophy, on the other hand, is a major cause of cardiomyocytes remodeling and HF. In addition to the transcriptional control of gene expression during hypertrophy, posttranscriptional regulation of protein expression is increasingly recognized as a vital mechanism for hypertrophic control (26) and m6A methyltransferase METTL3 is one of them (Table 1). Investigation showed that the level of $\mathrm{m} 6 \mathrm{~A}$ modification was significantly increased in isolated neonatal rat ventricular cardiomyocytes responded to hypertrophic stimulation by using $\mathrm{m} 6 \mathrm{~A}$ immunoprecipitation followed by RNA sequencing (27). In order to further investigate the possibility that $\mathrm{m} 6 \mathrm{~A}$ is involved in the regulation of cardiomyocyte homeostasis and hypertrophy in vivo, METTL3overexpressing mice were studied and showed significant cardiac hypertrophy when exposed to pressure overload stress. However, results did not show accelerated cardiac dysfunction. METTL3 knockout mice, on the other hand, revealed indications of failure on both morphological and functional levels (27). These evidences implied that the METTL3-m6A pathway could be a novel critical regulator of cardiac homeostasis. Interestingly, 
opposite findings were revealed by Kmietczyk et al. (28). In compared with healthy myocardium, their investigation indicated a higher METTL3 activity and a predominance of RNA transcripts enriched in $\mathrm{m} 6 \mathrm{~A}$ in human failing heart and dilated cardiomyopathy. Additionally, overexpression and knockdown of METTL3 expression affected the cellular size and cardiomyocytes remodeling both in vitro and in vivo. Mechanically, according to Dorn et al. (27), m6A peaks were selectively abundant in mRNAs encoding protein kinases and modifiers, such as mitogen-activated protein kinases (MAPKs) family, resulting in the significant increase of cardiomyocyte size. On the other side, Kmietczyk et al. (28) suggested the mRNA methylation is highly dynamic when exposed to stress conditions, which in turn to regulate translational efficiency by modifying transcript stability. These two findings appear to be contradictory, and previous researches have revealed that the genetic background of mice may influence the phenotypic outcome of cardiac disorders post-intervention. It is possible that variances in methodologies and mice strain backgrounds which may explain some of the disparities in the data. However, this also highlights that the underlying mechanisms are highly uncertain, and further investigations would be necessary.

As for FTO, a well-known m6A demethylase protein, has been linked to cardiac hypertrophy and muscular contraction in cardiac remodeling (Table 1). Gan et al. revealed that nuclear FTO expression in cardiomyocytes was considerably increased during an adipokine induced cardiomyocyte hypertrophic response in newborn rat cardiomyocytes, leptin-induced FTO upregulation in cardiomyocytes via JAK2/STAT3-dependent CUX1 upregulation (29). The researchers also discovered that FTO knockdown reduced the hypertrophic response in vitro, indicating that FTO plays an essential regulatory function in cardiac hypertrophy.

\section{Cardiomyocytes Death}

Recently, the role of apoptosis in the heart during ischemic and non-ischemic cardiomyopathies has been explored to involved in cardiac remodeling and heart failure. Structural or functional alterations in the myocardium may be intimately associated with myocardial ischemia. Alterations in blood supply that occur over time may result in changes in cardiac tissue which may have impacts on both short- and long-term cardiovascular function and prognosis. Myocardial infarction causes widespread cardiac damage via ischemic and ischemia/reperfusion injury, resulting in a deleterious effect on cardiomyocytes and cardiac functions. Cardiomyocyte apoptosis and necrosis, is a hallmark characteristic features of myocardial infarction and cardiac remodeling. Autophagy is triggered during ischemic stress to protect cardiomyocytes from ischemic or ischemia/reperfusion injury. However, excessive autophagy activation may have a deleterious effect on the heart in reperfusion and other stress circumstances, revealing the controversial nature of autophagy in myocardial ischemia. In numerous animal models, blocking apoptosis or regulating autophagy process could effectively reduce cardiac remodeling and heart failure $(30,31)$.

Song et al. (32) identified that in hypoxia/reoxygenation (H/R)-treated cardiomyocytes as well as the ischemia/reperfusion (I/R)-treated mice heart, the m6A methylations were increased owing to the upregulation of METTL3. Silencing METTL3 enhanced autophagic flux and inhibited apoptosis in $\mathrm{H} / \mathrm{R}$-treated cardiomyocytes. Whereas the RNA demethylase ALKBH5 acted in a opposite manner during myocardial I/R (32). Mechanistically, METTL3 methylates the transcription factor EB (TFEB), a critical regulator of lysosomal biogenesis and also stimulated the expression of autophagy genes (33). Silencing of METTL3 promoted TFEB expression and its nuclear translocation. Furthermore, TFEB can reduce METTL3 expression by decreasing the stability of the METTL3 mRNA. TFEB knockdown eliminated the enhanced autophagy driven by METTL3 downregulation, demonstrating that METTL3 mediates autophagic flux in a TFEB-dependent manner (32). Although authors did not evaluate changes to the overall m6A levels following ALKBH5 dysregulated, they demonstrated a vital role for $\mathrm{m} 6 \mathrm{~A}$ in autophagy regulation in $\mathrm{H} / \mathrm{R}$ cardiomyocytes (Table 1).

Besides, Mathiyalagan et al. (34) demonstrated the m6A modifications were increased in failing human (both ischemic and non-ischemic), post-MI pig and mouse hearts and hypoxic cardiomyocytes, resulting in a decrease in contractile performance. To identify the $\mathrm{m} 6 \mathrm{~A}$ regulators in the ischemic myocardium, the expressions of the major methylases, demethylases, and reader proteins were measured. They found FTO expression was significantly decreased in failing mammalian hearts and hypoxic cardiomyocytes. It is remarkable that loss of FTO exhibited a significantly increased number of arrhythmic events which are mediated primarily by cardiac contractile genes regulation, such as SERCA2A, MYH6/7 and RYR2, resulting in contractile dysfunction in cardiomyocytes. In vivo, FTO overexpression improved cardiac function remarkably during the chronic stage of postmyocardial infarction (34). FTO prevented cardiac contractile transcription deterioration and enhanced protein expression by selective demethylation under ischemic circumstances, thereby safeguarded cardiomyocyte contractile function by regulating intracellular $\mathrm{Ca}^{2+}$ dynamics (34).

\section{Extracellular Matrix Remodeling}

Cardiac fibrosis, defined by pathological activation of cardiac fibroblasts and excessive buildup of extracellular matrix (ECM) in the afflicted tissue. Cardiac fibroblasts are activated and transform into myofibroblasts which produce a large amount of matrix metalloproteinases (MMPs), leading to accumulation and composition alteration of ECM. In the cardiac remodeling, cardiac fibrosis contributes to the dysfunction of failing hearts by making the heart stiffer and reducing its function due to its inability to contract or conduct electric impulses required for the transmission of the contraction. The activation and differentiation of cardiac fibroblasts are also influenced by m6A methylation. Increased METTL3 expression was shown in cardiac fibroblasts treated with TGF- $\beta 1$ and in the murine hearts suffered from chronic myocardial infarction (35). METTL3 overexpression promoted cardiac fibrosis, fibroblastto-myofibroblast transition, and enhanced extracellular matrix production and accumulation, whereas METTL3 silencing had 
the opposite effect. In vivo, silencing METLL3 significantly suppressed cardiac fibrosis progression. Smad2/3 are key regulators of the fibrogenesis process in cardiac fibroblasts, whereas silencing METTL3 reduced the upregulation of $S \operatorname{mad} 2 / 3$ induced by TGF- $\beta 1$, suggesting that METTL3 regulated cardiac fibrosis at least partially through Smad-mediated pathway (35). Consistent with these results, Kmietczyk et al. (28) carefully analyzed and revealed that AAV9-mediated METTL3 overexpression following TAC operation significantly decreased fibrosis and collagen transcription in mice hearts. In addition to modifying cardiomyocyte hypertrophy, AAV9-mediated METTL3 overexpression may partially modulate the cardiac fibroblast phenotype. These evidences indicated METTL3mediated m6A methylation played a critical role in cardiac fibrosis regulation (Table 1).

In addition, along with the positively regulating contractile protein expression, FTO-dependent demethylation was also indicated to affected critical non-contractile processes involving tissue morphogenesis, angiogenesis, extracellular matrix organization, fibrosis, and cell proliferation and differentiation in murine MI hearts (34). In FTO-overexpressing mice post-MI models, fibrosis in mice overexpressing FTO was significantly decreased compared with their respective controls, as measured by scar size (\%) (34).

\section{Vascular Remodeling}

Vascular remodeling refers to alterations in the structure of resistance vessels contributing to elevated systemic vascular resistance. It is a dynamic structural change that involves alterations to numerous cellular including endothelial cells (ECs), vascular smooth muscle cells (VSMCs), and fibroblasts and non-cellular components, which may lead to vascular stiffness. During vascular remodeling and heart failure, abnormalities in ECs and VSMCs proliferation and migration, endothelial dysfunction, inflammatory processes, and the production or breakdown of extracellular matrix components are present and predominant $(36,37)$. METTL3 and FTO have been shown to be involved in the regulation of vascular or cardiac dysfunction under stress conditions (Table 1). Under hypoxia stimulations, METTL3 can stimulate the differentiation of adipose-derived stem cells into vascular VSMCs (38). Also, evidences showed that overexpression of FTO in the ischemic myocardium could reduce cardiac fibrosis and increase angiogenesis (34). For angiogenesis, anti-CD31 immunostaining was performed by Mathiyalagan et al. (34). They measured the angiogenic response by detecting CD31-positive endothelial cells in the infarct border zone of murine hearts 4 weeks after MI, and discovered FTOoverexpressing hearts had a much higher proportion of CD31positive cells than their respective control group (34). METTL14, as a methylase, was discovered to stimulate inflammatory response in ECs and promote atherosclerotic plaque formation by interacting with forkhead box O1 (FOXO1). Through YTHDF1 recognition, METTL14 enhanced the translation of FOXO1 mRNA, therefore increased the expression of the endothelial adhesion molecules VCAM-1 and ICAM-1, which promoting endothelial adhesion (39). In addition, researchers revealed that METTL14 mediated the m6A modification of
pri-miR-19a, promoted its processing and maturation, which promoting the invasion and proliferation of cardiovascular endothelial cells (8).

\section{LncRNAs in Cardiac Remodeling}

Numerous investigations have revealed that long noncoding RNAs played critical regulatory roles under stressful situation: lncRNAs Mhrt, XIST, MIAT, Chast, CHRF, ROR, H19, and Plscr4 are involved in myocardial hypertrophy, whereas XIST, MALAT1, GAS5, Neat1, AK139328, APF, CAIF, AK088388, CARL, HOTAIR, and NRF are involved in cardiomyocytes apoptosis and necrosis. Moreover, MIAT, MALAT1, Wisper, MEG3, and H19 are involved in extracellular matrix reconstruction. In addition, MALAT1, GAS5, H19, TUG1, AK098656, TRPV1, Giver, and Lnc-Ang362 have been implicated in vascular remodeling $(11,40)$. m6A modification has been identified in long non-coding RNAs by methylated RNA immunoprecipitation sequencing (MeRIP-seq) m6A on lncRNAs accounts for $\sim 12 \%$ of the total m6A peaks (41). The m6A methylation of lncRNAs has been demonstrated to modify their structure (42), stability (43), transport and destruction (44), hence altering the biological processes or the interaction with other RNA molecules (45-47). However, only a few of m6A modification on lncRNA have been reported in cardiac diseases. In this section, we will focus on the potential function of m6A of lncRNAs in cardiac remodeling (Table 2).

LncRNA myosin heavy chain associated RNA transcript (Mhrt) have been proven in regulating the cardiac remodeling. Mhrt is related to heart failure due to its ability to modulate cardiac hypertrophy. Mhrt increased the expression of KLF4 through direct binding to miR-145a-5p or inhibiting phosphorylation of KLF4, to prevent ERK and KLF4 interaction, hence inhibiting myocardin expression and the development of cardiac hypertrophy (48). Besides, Mhrt could protect cardiomyocytes from apoptosis against the $\mathrm{H}_{2} \mathrm{O}_{2}$ or $\mathrm{H} / \mathrm{R}$ exposure (49). Shen et al. (50) observed that the expression of FTO and Mhrt were downregulated in heart failure mouse model. FTO overexpression inhibited the m6A modification of Mhrt, hence increased the expression of Mhrt, which inhibited the apoptosis of cardiomyocytes induced by the H/R. These results indicated that m6A modification of Mhrt participates in the development of cardiac disease (50).

LncRNA X-inactive specific transcript (XIST), plays a critical role in the regulation throughout the whole spectrum of human diseases and can be utilized as a novel diagnostic and prognostic biomarker for human disease $(51,52)$. XIST was investigated positively regulates S100B expression and enhance TLR2 expression, thereby modulates the progression of cardiomyocyte hypertrophy by miR-330-3p/S100B pathway and miR-101/TLR2 axis $(53,54)$. XIST was up-regulated in cardiomyocytes after infarction. Overexpression of XIST might promote cardiomyocyte apoptosis and inhibit proliferation by mediating PDE4D expression via targeting miR-130a-3p (55). In acute myocardial infarction, XIST protected hypoxia-induced cardiomyocyte injury and repressed myocardial apoptosis by interacting directly with various miRNAs and positively regulated expression of anti-apoptotic biomarkers such as Bax, 
TABLE 2 | Potential role of IncRNA m6A modification in cardiac remodeling.

\begin{tabular}{|c|c|c|c|}
\hline LncRNAs & LncRNAs in cardiac remodeling & m6A regulators & LncRNAs modified with m6A \\
\hline \multirow[t]{2}{*}{ Mhrt } & $\begin{array}{l}\text { Mhrt regulated the expression of KLF4 to prevent } \\
\text { ERK and KLF4 interaction, hence inhibiting the } \\
\text { development of cardiac hypertrophy (48). }\end{array}$ & FTO & $\begin{array}{l}\text { FTO downregulated in heart failure mouse model. FTO } \\
\text { overexpression increased the expression of Mhrt, which } \\
\text { inhibited the apoptosis of cardiomyocytes induced by } \\
\text { the H/R (50). }\end{array}$ \\
\hline & $\begin{array}{l}\text { Mhrt protects cardiomyocytes from apoptosis } \\
\text { against the } \mathrm{H}_{2} \mathrm{O}_{2} \text { or } \mathrm{H} / \mathrm{R} \text { exposure (49). }\end{array}$ & & \\
\hline \multirow[t]{3}{*}{ XIST } & $\begin{array}{l}\text { XIST could modulate the progression of } \\
\text { cardiomyocyte hypertrophy by regulating } \\
\text { miR-330-3p/S100B pathway and miR-101/TLR2 } \\
\text { axis }(53,54) \text {. }\end{array}$ & $\begin{array}{l}\text { RBM15/ } \\
\text { RBM15B, WTAP, METTL3 }\end{array}$ & $\begin{array}{l}\text { XIST is highly methylated with at least } 78 \mathrm{~m} 6 \mathrm{~A} \\
\text { residues (45). } \\
\text { m6A methylation of XIST creates a multiprotein complex } \\
\text { and recruits the silencing complex, thereby promotes } \\
\text { XIST-mediated transcriptional repression (45). }\end{array}$ \\
\hline & $\begin{array}{l}\text { Overexpression of XIST promoted cardiomyocyte } \\
\text { apoptosis and inhibit proliferation by mediating } \\
\text { PDE4D expression via targeting miR-130a-3p (55). }\end{array}$ & & \\
\hline & $\begin{array}{l}\text { In acute myocardial infarction, XIST regulated } \\
\text { expression of anti-apoptotic biomarkers Bax, } \\
\text { hexokianse } 2 \text { and Notch1 }(56,57) \text {. }\end{array}$ & & \\
\hline \multirow[t]{3}{*}{ MALAT1 } & $\begin{array}{l}\text { MALAT1 could increase cardiomyocytes } \\
\text { proliferation or apoptosis in myocardial I/R rats } \\
\text { through activating PI3K/Akt and } \beta \text {-catenin signaling } \\
\text { pathways }(59,60) \text {. }\end{array}$ & / & $\begin{array}{l}\text { SCARLET verified MALAT1 was also highly modified by } \\
\text { m6A and contained several m6A motifs (A2515, A2577, } \\
\text { A2611, and A2720) (66). }\end{array}$ \\
\hline & $\begin{array}{l}\text { MALAT1 promoted cardiomyocyte apoptosis of } \\
\text { HL-1 or H9c2 cells under H/R conditions via } \\
\text { interacting with microRNAs }(61,62) \text {. }\end{array}$ & HNRNPC and HNRNPG & $\begin{array}{l}\text { The binding of MALAT1 and the m6A "reader" HNRNPC } \\
\text { and HNRNPG would increase if A2577 and A2515 of } \\
\text { MALAT1 modified by m6A, thereby alter the expression } \\
\text { of MALAT1(67). }\end{array}$ \\
\hline & $\begin{array}{l}\text { MALAT1 promoted human endothelial cells } \\
\text { pyroptosis by affecting NLRP3 expression through } \\
\text { competitively binding miR-22 (63). } \\
\text { MALAT1 mediated cardiac fibrosis in MI mice } \\
\text { model (64). }\end{array}$ & & \\
\hline \multirow[t]{2}{*}{ GAS5 } & $\begin{array}{l}\text { GAS5 hasten myocardial I/R injury by sponging } \\
\text { miR-532-5p in myocardial cells (68). }\end{array}$ & ALKBH5, YTHDF2 & $\begin{array}{l}\text { ALKBH5 inhibited m6A modification of GAS5 to its } \\
\text { stability. Also, m6A promoted the degradation of GAS5 } \\
\text { in a YTHDF2-dependent manner (70). }\end{array}$ \\
\hline & $\begin{array}{l}\text { GAS5 knockdown would aggravate microvascular } \\
\text { dysfunction by altering } \beta \text {-catenin signaling activity } \\
\text { (69). }\end{array}$ & YTHDF3 & $\begin{array}{l}\text { Silencing m6A "reader" YTHDF3 enhances the } \\
\text { degradation of GAS5 (44). }\end{array}$ \\
\hline $\mathrm{H} 19$ & $\begin{array}{l}\text { H19 promoted myocardial apoptosis (71). } \\
\text { H19 overexpression promoted VSMCs proliferation } \\
\text { and inhibited its apoptosis (72). }\end{array}$ & ALKBH5 & $\begin{array}{l}\text { ALKBH5 regulated the expression of } \mathrm{H} 19 \text { by mediating } \\
\text { its m6A modification levels in } \mathrm{H} 9 \mathrm{c} 2 \text { cells with } \\
\mathrm{H}_{2} \mathrm{O}_{2} \text {-induced senescence (73). }\end{array}$ \\
\hline
\end{tabular}

hexokianse 2, and Notch1 $(56,57)$. Researchers have shown XIST is highly methylated with at least $78 \mathrm{~m} 6 \mathrm{~A}$ residues. m6A methylation of XIST creates a multiprotein complex with RBM15/RBM15B, WTAP, and METTL3, which in turn recruits the silencing complex, thereby promotes XIST-mediated transcriptional repression (45).

LncRNA metastasis-associated lung adenocarcinoma transcript 1 (MALAT1) is widely expressed and highly conserved in human tissues (58). MALAT1 has been reported to increase cardiomyocytes proliferation or apoptosis in rats with myocardial I/R induced injury through activating canonical signaling pathways, such as PI3K/Akt and $\beta$-catenin signaling pathways $(59,60)$. Also, MALAT1 was discovered to promote cardiomyocyte apoptosis of HL-1 or H9C2 cells under H/R conditions via interacting with microRNAs $(61,62)$. LncRNA MALAT1 profoundly induced by stimuli such as hypoxia, cytokine and oxidative stress in ECs, and it could regulate various pathophysiological processes of cardiac remodeling regarding to vascular remodeling $(63,64)$. For example, ox-LDL is thought to be a critical factor in the initiation and progression of endothelial dysfunction. MALAT1 induced by ox-LDL was also found to protect against endothelial injury by sponging the miR-22-3p via activation of AKT pathway (65). Recently, several m6A-deposition sites of MALAT1 have been identified, by the method which can accurately determines m6A status at any site in mRNA/lncRNA, named SCARLET. It has been verified that lncRNA MALAT1 was also highly modified by m6A and contained several m6A motifs (A2515, A2577, A2611, and A2720) (66). m6A modifications on site A2577 and A2515 of MALAT1 destabilize the RNA hairpin of MALAT1, releasing the poly- $U$ tract and increasing the binding with HNRNPC and HNRNPG $(42,67)$, indicating MALAT1 could be m6A modified to regulate the development of various diseases including cardiac remodeling. 
LncRNA GAS5 was found to promote myocardial I/R injury by sponging miR-532-5p in myocardial cells (68). LncRNA GAS5 was also confirmed to participate in the vascular remodeling. Wang et al. showed that lncRNA GAS5 regulated ECs and VSMCs function, GAS5 knockdown would aggravate microvascular dysfunction by altering $\beta$-catenin signaling activity, thus increased neovascularization and capillary leakage (69). The m6A modifications of GAS5 have been found. ALKBH5 inhibited m6A modification of GAS5 to its stability. Besides this, m6A promoted the degradation of GAS5 in a YTHDF2dependent manner (70). Additionally, it was discovered that silencing $\mathrm{m} 6 \mathrm{~A}$ "reader" YTHDF3 enhances the degradation of GAS5 (44). These findings suggests that m6A-modified GAS5 may play a role in the development of cardiac remodeling.

In recent decades, IncRNA H19, a potential serum marker for coronary heart disease, is involved in the regulation of vascular remodeling. For example, in I/R condition, lncRNA H19 is reported to largely participated and promoted myocardial apoptosis (71). Researchers have investigated that H19 overexpression promoted VSMCs proliferation and inhibited its apoptosis (72). The m6A modified-H19 has been revealed involving in the development of cardiac diseases. In $\mathrm{H}_{2} \mathrm{O}_{2}$ induced senescence, lncRNA H19 expression decreased and m6A modification increased following $\mathrm{H} / \mathrm{R}$, ALKBH5 regulated the expression of $\mathrm{H} 19$ by mediating its m6A modification levels (73).

\section{CONCLUSIONS AND FUTURE PROSPECTS}

In cardiac remodeling which is the fundamental step for the progression of heart failure, $\mathrm{m} 6 \mathrm{~A}$ modification shows great potential in mechanistic studies and therapeutic target

\section{REFERENCES}

1. Wang Y, Wang C, Ma J. Role of cardiac endothelial cells-derived micrornas in cardiac remodeling. Discov Med. (2019) 28:95-105.

2. Handy DE, Castro R, Loscalzo J. epigenetic modifications: basic mechanisms and role in cardiovascular disease. Circulation. (2011) 123:2145-56. doi: 10.1161/CIRCULATIONAHA.110.956839

3. Lu D, Thum T. RNA-based diagnostic and therapeutic strategies for cardiovascular disease. Nat Rev Cardiol. (2019) 16:661-74. doi: 10.1038/s41569-019-0218-x

4. Frye M, Harada BT, Behm M, He C. RNA modifications modulate gene expression during development. Science. (2018) 361:1346-9. doi: 10.1126/science.aau1646

5. Li Z, Zhao P, Xia Q. Epigenetic methylations on n6-adenine and n6-adenosine with the same input but different output. Int J Mol Sci. (2019) 20:2931. doi: 10.3390/ijms20122931

6. Roignant J-Y, Soller M. m6A in mRNA: an ancient mechanism for fine-tuning gene expression. Trends Genetics. (2017) 33:380-90. doi: 10.1016/j.tig.2017.04.003

7. Lin S, Choe J, Du P, Triboulet R, Gregory RI. The m6A methyltransferase METTL3 promotes translation in human cancer cells. Mol Cell. (2016) 62:335-45. doi: 10.1016/j.molcel.2016.03.021

8. Zhang B-Y, Han L, Tang Y-F, Zhang G-X, Fan X-L, Zhang J-J, et al. METTL14 regulates M6A methylation-modified primary mir-19a to promote explorations. However, m6A has complicated impacts on gene expression and is hard to be isolated at a cellular level. It is hard to totally remove the complex of m6A methyltransferase, as knockout typically results in cell death. Studies were significantly impeded by this situation. It will require more studies to completely illustrate how m6A impacts the in vivo behavior of target RNAs and its direct downstream effects on gene expression in the setting of cardiac remodeling.

It is worth noting that, the interplay of m6A and lncRNAs is prevalent and inspiring, which significantly broadening the scope of epitranscriptomic regulation. m6A modifying lncRNAs still needs to interact with other components, such as other proteins, miRNAs and mRNAs. m6A modification on lncRNAs are emerging regulators in diverse process but remained in the level of phenotype researches rather than further mechanism manifestations. In addition, it remains unknown how m6A modifications on lncRNAs differs from the those on mRNAs in cardiac remodeling. As a whole, improvements in RNA modification sequencing and mapping technologies will definitely lead to a deeper understanding of RNA epigenetics mechanisms, and encourages the development of novel molecular treatments for cardiac remodeling in the future.

\section{AUTHOR CONTRIBUTIONS}

MC and RX wrote the manuscript. YW drew the figure. WF, YD, and CL edited the manuscript. All authors contributed to the article and approved the submitted version.

\section{FUNDING}

The present study was funded by the National Natural Science Foundation of China (No. 81770394). cardiovascular endothelial cell proliferation and invasion. Eur Rev Med Pharmacol Sci. (2020) 24:7015-23. doi: 10.26355/eurrev_202006_21694

9. Zhang B, Xu Y, Cui X, Jiang H, Luo W, Weng X, et al. Alteration of m6A RNA methylation in heart failure with preserved ejection fraction. Front Cardiovasc Med. (2021) 8:647806. doi: 10.3389/fcvm.2021.647806

10. Bridges MC, Daulagala AC, Kourtidis A. LNCcation: IncRNA localization and function. J Cell Biol. (2021) 220:e202009045. doi: 10.1083/jcb.202009045

11. Zhou H, Wang B, Yang Y-X, Jia Q-J, Zhang A, Qi Z-W, et al. Long non-coding RNAS in pathological cardiac remodeling: a review of the update literature. Biomed Res Int. (2019) 2019:7159592. doi: 10.1155/2019/7159592

12. Kazimierczyk M, Wrzesinski J. Long non-coding RNA epigenetics. Int J Mol Sci. (2021) 22:6166. doi: 10.3390/ijms22116166

13. Ma S, Chen C, Ji X, Liu J, Zhou Q, Wang G, et al. The interplay between m6A RNA methylation and non-coding RNA in cancer. J Hematol Oncol. (2019) 12:121. doi: 10.1186/s13045-019-0805-7

14. Zhou KI, Pan T. Structures of the m6A methyltransferase complex: two subunits with distinct but coordinated roles. Mol Cell. (2016) 63:183-5. doi: 10.1016/j.molcel.2016.07.005

15. Little NA, Hastie ND, Davies RC. Identification of WTAP, a novel Wilms' tumour 1-associating protein. Human Mol Genetics. (2000) 9:2231-9. doi: 10.1093/oxfordjournals.hmg.a018914

16. Garcias Morales D, Reyes JL. A birds'-eye view of the activity and specificity of the mrna m6 a methyltransferase complex. Wiley Interdiscip Rev RNA. (2021) 12:e1618. doi: $10.1002 /$ wrna. 1618 
17. Jia G, Fu Y, Zhao X, Dai Q, Zheng G, Yang Y, et al. N6-methyladenosine in nuclear RNA is a major substrate of the obesity-associated FTO. Nat Chem Biol. (2011) 7:885-7. doi: 10.1038/nchembio.687

18. Zheng G, Dahl JA, Niu Y, Fedorcsak P, Huang C-M, Li CJ, et al. ALKBH5 is a mammalian RNA demethylase that impacts RNA metabolism and mouse fertility. Mol Cell. (2013) 49:18-29. doi: 10.1016/j.molcel.2012.10.015

19. Cao G, Li H-B, Yin Z, Flavell RA. Recent advances in dynamic m6A RNA modification. Open Biol. 6:160003. doi: 10.1098/rsob.160003

20. Yu F, Wei J, Cui X, Yu C, Ni W, Bungert J, et al. Post-translational modification of RNA m6A demethylase ALKBH5 regulates ROS-induced DNA damage response. Nucleic Acids Res. (2021) 49:5779-97. doi: 10.1093/nar/gkab415

21. Berlivet S, Scutenaire J, Deragon J-M, Bousquet-Antonelli C. Readers of the m6A epitranscriptomic code. Biochimica et Biophysica Acta (BBA) - Gene Regulat Mechan. (2019) 1862:329-42. doi: 10.1016/j.bbagrm.2018.12.008

22. Bell JL, Wächter K, Mühleck B, Pazaitis N, Köhn M, Lederer M, et al. Insulin-like growth factor 2 MRNA-binding proteins (IGF2BPS): posttranscriptional drivers of cancer progression? Cell Mol Life Sci. (2013) 70:2657-75. doi: 10.1007/s00018-012-1186-z

23. Alarcón CR, Goodarzi H, Lee H, Liu X, Tavazoie S, Tavazoie SF. Hnrnpa2b1 is a mediator of m(6)a-dependent nuclear RNA processing Events. Cell. (2015) 162:1299-308. doi: 10.1016/j.cell.2015.08.011

24. Wolf DA, Lin Y, Duan H, Cheng Y. eIF-Three to tango: emerging functions of translation initiation factor eIF3 in protein synthesis and disease. J Mol Cell Biol. (2020) 12:403-9. doi: 10.1093/jmcb/mjaa018

25. Berulava T, Buchholz E, Elerdashvili V, Pena T, Islam MR, Lbik D, et al. Changes in m6A RNA methylation contribute to heart failure progression by modulating translation. Eur J Heart Fail. (2020) 22:54-66. doi: 10.1002/ejhf.1672

26. Hinger SA, Wei J, Dorn LE, Whitson BA, Janssen PML, He C, et al. Remodeling of the m6A landscape in the heart reveals few conserved posttranscriptional events underlying cardiomyocyte hypertrophy. J Mol Cell Cardiol. (2021) 151:46-55. doi: 10.1016/j.yjmcc.2020.11.002

27. Dorn LE, Lasman L, Chen J, Xu X, Hund TJ, Medvedovic M, et al. The N6-methyladenosine mRNA methylase METTL3 controls cardiac homeostasis and hypertrophy. Circulation. (2019) 139:533-45. doi: 10.1161/CIRCULATIONAHA.118.036146

28. Kmietczyk V, Riechert E, Kalinski L, Boileau E, Malovrh E, Malone $\mathrm{B}$, et al. m6A-mRNA methylation regulates cardiac gene expression and cellular growth. Life Science Alliance. (2019) 2:e201800233. doi: 10.26508/lsa.201800233

29. Gan XT, Zhao G, Huang CX, Rowe AC, Purdham DM, Karmazyn M. Identification of fat mass and obesity associated (FTO) protein expression in cardiomyocytes: regulation by leptin and its contribution to leptin-induced hypertrophy. PLOS ONE. (2013) 8:e74235. doi: 10.1371/journal.pone.0074235

30. Yang R, Song Z, Wu S, Wei Z, Xu Y, Shen X. Toll-like receptor 4 contributes to a myofibroblast phenotype in cardiac fibroblasts and is associated with autophagy after myocardial infarction in a mouse model. Atherosclerosis. (2018) 279:23-31. doi: 10.1016/j.atherosclerosis.2018.10.018

31. Xiong R, Li N, Chen L, Wang W, Wang B, Jiang W, et al. STING protects against cardiac dysfunction and remodelling by blocking autophagy. Cell Commun Signal. (2021) 19:109. doi: 10.1186/s12964-021-00793-0

32. Song H, Feng X, Zhang H, Luo Y, Huang J, Lin M, et al. METTL 3 and ALKBH5 oppositely regulate $\mathrm{m} 6 \mathrm{~A}$ modification of TFEB mRNA, which dictates the fate of hypoxia/reoxygenation-treated cardiomyocytes. Autophagy. (2019) 15:1419-37. doi: 10.1080/15548627.2019.1586246

33. Zhao E, Czaja MJ. TFEB: a central regulator of both the autophagosome and lysosome. Hepatology. (2012) 55:1632-4. doi: 10.1002/hep.25619

34. Mathiyalagan P, Adamiak M, Mayourian J, Sassi Y, Liang Y, Agarwal $\mathrm{N}$, et al. FTO-dependent n6-methyladenosine regulates cardiac function during remodeling and repair. Circulation. (2019) 139:518-32. doi: 10.1161/CIRCULATIONAHA.118.033794

35. Li T, Zhuang Y, Yang W, Xie Y, Shang W, Su S, et al. Silencing of METTL3 attenuates cardiac fibrosis induced by myocardial infarction via inhibiting the activation of cardiac fibroblasts. FASEB J. (2021) 35:e21162. doi: 10.1096/fj.201903169R

36. Cai Z, Gong Z, Li Z, Li L, Kong W. Vascular extracellular matrix remodeling and hypertension. Antioxid Redox Signal. (2021) 34:765-83. doi: $10.1089 /$ ars.2020.8110
37. Renna NF, de las Heras N, Miatello RM. Pathophysiology of vascular remodeling in hypertension. Int J Hypertens. (2013) 2013:808353. doi: $10.1155 / 2013 / 808353$

38. Lin J, Zhu Q, Huang J, Cai R, Kuang Y. Hypoxia promotes vascular smooth muscle cell (VSMC) differentiation of adipose-derived stem cell (ADSC) by regulating mettl3 and paracrine factors. Stem Cells Int. (2020) 2020:2830565. doi: 10.1155/2020/2830565

39. Jian D, Wang Y, Jian L, Tang H, Rao L, Chen K, et al. METTL14 aggravates endothelial inflammation and atherosclerosis by increasing FOXO1 N6-methyladeosine modifications. Theranostics. (2020) 10:8939-56. doi: $10.7150 /$ thno.45178

40. Poller W, Dimmeler S, Heymans S, Zeller T, Haas J, Karakas M, et al. Non-coding RNAs in cardiovascular diseases: diagnostic and therapeutic perspectives. Eur Heart J. (2018) 39:2704-16. doi: 10.1093/eurheartj/ehx165

41. Cui X, Meng J, Rao MK, Chen Y, Huang Y. HEPeak: an HMM-based exome peak-finding package for RNA epigenome sequencing data. BMC Genomics. (2015) 16:S2. doi: 10.1186/1471-2164-16-S4-S2

42. Liu N, Dai Q, Zheng G, He C, Parisien M, Pan T. N(6)-methyladenosinedependent RNA structural switches regulate RNA-protein interactions. Nature. (2015) 518:560-4. doi: 10.1038/nature14234

43. Brown JA, Kinzig CG, DeGregorio SJ, Steitz JA. Methyltransferase-like protein 16 binds the 3'-terminal triple helix of MALAT1 long non-coding RNA. Proc Natl Acad Sci U S A. (2016) 113:14013-8. doi: 10.1073/pnas.1614759113

44. Ni W, Yao S, Zhou Y, Liu Y, Huang P, Zhou A, et al. Long non-coding RNA GAS5 inhibits progression of colorectal cancer by interacting with and triggering YAP phosphorylation and degradation and is negatively regulated by the m6A reader YTHDF3. Mol Cancer. (2019) 18:143. doi: 10.1186/s12943-019-1079-y

45. Patil DP, Chen C-K, Pickering BF, Chow A, Jackson C, Guttman M, et al. $\mathrm{m}(6)$ A RNA methylation promotes XIST-mediated transcriptional repression. Nature. (2016) 537:369-73. doi: 10.1038/nature19342

46. Jin D, Guo J, Wu Y, Du J, Yang L, Wang X, et al. m6A mRNA methylation initiated by METTL3 directly promotes YAP translation and increases YAP activity by regulating the MALAT1-miR-1914-3p-YAP axis to induce NSCLC drug resistance and metastasis. J Hematol Oncol. (2019) 12:135. doi: 10.1186/s13045-019-0830-6

47. Zheng Z-Q, Li Z-X, Zhou G-Q, Lin L, Zhang L-L, Lv J-W, et al. Long non-coding RNA FAM225A promotes nasopharyngeal carcinoma tumorigenesis and metastasis by acting as ceRNA to Sponge miR-5903p/miR-1275 and upregulate ITGB3. Cancer Res. (2019) 79:4612-26. doi: 10.1158/0008-5472.CAN-19-0799

48. Xu Y, Luo Y, Liang C, Zhang T. LncRNA-Mhrt regulates cardiac hypertrophy by modulating the miR-145a-5p/KLF4/myocardin axis. J Mol Cell Cardiol. (2020) 139:47-61. doi: 10.1016/j.yjmcc.2019.12.013

49. Zhang J, Gao C, Meng M, Tang H. Long non-coding RNA MHRT Protects cardiomyocytes against H2O2-induced apoptosis. Biomol Ther (Seoul). (2016) 24:19-24. doi: 10.4062/biomolther.2015.066

50. Shen W, Li H, Su H, Chen K, Yan J. FTO overexpression inhibits apoptosis of hypoxia/reoxygenation-treated myocardial cells by regulating m6A modification of Mhrt. Mol Cell Biochem. (2021) 476:2171-9. doi: 10.1007/s11010-021-04069-6

51. Patrat C, Ouimette J-F, Rougeulle C. X chromosome inactivation in human development. Development. (2020) 147:dev183095. doi: 10.1242/dev. 183095

52. Liu X, Ming X, Jing W, Luo P, Li N, Zhu M, et al. Long non-coding RNA XIST predicts worse prognosis in digestive system tumors: a systemic review and meta-analysis. Biosci Rep. (2018) 38:BSR20180169. doi: 10.1042/ BSR20180169

53. Chen Y, Liu X, Chen L, Chen W, Zhang Y, Chen J, et al. The long non-coding RNA XIST protects cardiomyocyte hypertrophy by targeting miR-330-3p. Biochem Biophys Res Commun. (2018) 505:807-15. doi: 10.1016/j.bbrc.2018.09.135

54. Xiao L, Gu Y, Sun Y, Chen J, Wang X, Zhang Y, et al. The long non-coding RNA XIST regulates cardiac hypertrophy by targeting mir-101. J Cell Physiol. (2019) 234:13680-92. doi: 10.1002/jcp.28047

55. Zhou T, Qin G, Yang L, Xiang D, Li S. LncRNA XIST regulates myocardial infarction by targeting mir-130a-3p. J Cell Physiol. (2019) 234:8659-67. doi: $10.1002 /$ jcp. 26327 
56. Zhou J, Li D, Yang B-P, Cui W-J. LncRNA XIST inhibits hypoxiainduced cardiomyocyte apoptosis via mediating mir-150-5p/bax in acute myocardial infarction. Eur Rev Med Pharmacol Sci. (2020) 24:1357-66. doi: 10.26355/eurrev_202002_20193

57. Fan J-L, Zhu T-T, Xue Z-Y, Ren W-Q, Guo J-Q, Zhao H-Y, et al. IncRNA-XIST protects the hypoxia-induced cardiomyocyte injury through regulating the miR-125b-hexokianse 2 axis. In vitro Cell Dev Biol Anim. (2020) 56:349-57. doi: 10.1007/s11626-020-00459-0

58. Ma X-Y, Wang J-H, Wang J-L, Ma CX, Wang X-C, Liu F-S. Malat1 as an evolutionarily conserved lncRNA, plays a positive role in regulating proliferation and maintaining undifferentiated status of early-stage hematopoietic cells. BMC Genomics. (2015) 16:676. doi: 10.1186/s12864-015-1881-x

59. Sun T, Cheng Y-T, Yan L-X, Krittanawong C, Qian W, Zhang HJ. LncRNA MALAT1 knockdown alleviates myocardial apoptosis in rats with myocardial ischemia-reperfusion through activating PI3K/AKT signaling pathway. Eur Rev Med Pharmacol Sci. (2019) 23:10523-31. doi: 10.26355/eurrev_201912_19693

60. Xu X-Z, Luo B, Xiao Y, Zheng W-Q. Effects of IncRNA MALAT1mediated $\beta$-catenin signaling pathway on myocardial cell apoptosis in rats with myocardial ischemia/reperfusion injury. Eur Rev Med Pharmacol Sci. (2019) 23:9557-65. doi: 10.26355/eurrev_201911_19450

61. Gong $\mathrm{X}$, Zhu Y, Chang $\mathrm{H}$, Li Y, Ma F. Long non-coding RNA MALAT1 promotes cardiomyocyte apoptosis after myocardial infarction via targeting miR-144-3p. Biosci Rep. (2019) 39: BSR20191103. doi: 10.1042/BSR20191103

62. Wang C, Liu G, Yang H, Guo S, Wang H, Dong Z, et al. MALAT1-mediated recruitment of the histone methyltransferase EZH2 to the microRNA-22 promoter leads to cardiomyocyte apoptosis in diabetic cardiomyopathy. Sci Total Environ. (2021) 766:142191. doi: 10.1016/j.scitotenv.2020.142191

63. Song Y, Yang L, Guo R, Lu N, Shi Y, Wang X. Long non-coding RNA MALAT1 promotes high glucose-induced human endothelial cells pyroptosis by affecting NLRP3 expression through competitively binding mir-22. Biochem Biophys Res Commun. (2019) 509:359-66. doi: 10.1016/j.bbrc.2018.12.139

64. Huang S, Zhang L, Song J, Wang Z, Huang X, Guo Z, et al. Long non-coding RNA MALAT1 mediates cardiac fibrosis in experimental postinfarct myocardium mice model. J Cell Physiol. (2019) 234:2997-3006. doi: $10.1002 / j c p .27117$

65. Tang Y, Jin X, Xiang Y, Chen Y, Shen C, Zhang Y, et al. The IncRNA MALAT1 protects the endothelium against ox-LDL-induced dysfunction via upregulating the expression of the miR-22-3p target genes CXCR2 and AKT. FEBS Lett. (2015) 589(20 Pt B):3189-96. doi: 10.1016/j.febslet.2015.08.046

66. Liu N, Parisien M, Dai Q, Zheng G, He C, Pan T. Probing N6-methyladenosine RNA modification status at single nucleotide resolution in mrna and long non-coding RNA. RNA. (2013) 19:1848-56. doi: 10.1261/rna.041178.113
67. Liu N, Zhou KI, Parisien M, Dai Q, Diatchenko L, Pan T. N6-methyladenosine alters RNA structure to regulate binding of a low-complexity protein. Nucleic Acids Research. (2017) 45:6051-63. doi: 10.1093/nar/gkx141

68. Han Y, Wu N, Xia F, Liu S, Jia D. Long non-coding RNA GAS5 regulates myocardial ischemia-reperfusion injury through the PI3K/AKT apoptosis pathway by sponging miR-532-5p. Int J Mol Med. (2020) 45:858-72. doi: 10.3892/ijmm.2020.4471

69. Wang Y-N-Z, Shan K, Yao M-D, Yao J, Wang J-J, Li X, et al. Long non-coding RNA-GAS5: a novel regulator of hypertensioninduced vascular remodeling. Hypertension. (2016) 68:736-48. doi: 10.1161/HYPERTENSIONAHA.116.07259

70. Wang X, Zhang J, Wang Y. Long non-coding RNA GAS5-AS1 suppresses growth and metastasis of cervical cancer by increasing GAS5 stability. Am J Transl Res. (2019) 11:4909-21.

71. Luo H, Wang J, Liu D, Zang S, Ma N, Zhao L, et al. The lncRNA H19/miR675 axis regulates myocardial ischemic and reperfusion injury by targeting PPAR $\alpha$. Mol Immunol. (2019) 105:46-54. doi: 10.1016/j.molimm.2018.11. 011

72. Zhang L, Cheng H, Yue Y, Li S, Zhang D, He R. H19 knockdown suppresses proliferation and induces apoptosis by regulating $\mathrm{miR}-148 \mathrm{~b} / \mathrm{WNT} / \beta$-catenin in ox-LDL -stimulated vascular smooth muscle cells. J Biomed Sci. (2018) 25:11. doi: 10.1186/s12929-018-0418-4

73. Zhang X, Fu Q, Xu L, Yang Y, Zhao W, Zhang Y, et al. Dexmedetomidine postconditioning alleviates hypoxia/reoxygenation injury in senescent myocardial cells by regulating lncRNA H19 and m6A modification. Oxidative Med Cell Longevity. (2020) 2020:e9250512. doi: 10.1155/2020/9250512

Conflict of Interest: The authors declare that the research was conducted in the absence of any commercial or financial relationships that could be construed as a potential conflict of interest.

Publisher's Note: All claims expressed in this article are solely those of the authors and do not necessarily represent those of their affiliated organizations, or those of the publisher, the editors and the reviewers. Any product that may be evaluated in this article, or claim that may be made by its manufacturer, is not guaranteed or endorsed by the publisher.

Copyright (C) 2022 Choy, Xue, Wu, Fan, Dong and Liu. This is an open-access article distributed under the terms of the Creative Commons Attribution License (CC BY). The use, distribution or reproduction in other forums is permitted, provided the original author(s) and the copyright owner(s) are credited and that the original publication in this journal is cited, in accordance with accepted academic practice. No use, distribution or reproduction is permitted which does not comply with these terms. 